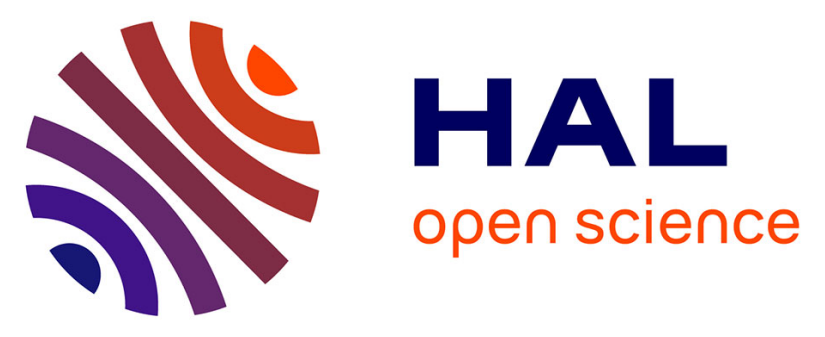

\title{
First landscape of binding to chromosomes for a domesticated mariner transposase in the human genome: diversity of genomic targets of SETMAR isoforms in two colorectal cell lines
}

\author{
Aymeric Antoine-Lorquin, Ahmed Arnaoty, Sassan Asgari, Martine Batailler, \\ Linda Beauclair, Catherine Belleannée, Solenne Bire, Nicolas Buisine, Vincent \\ Coustham, Alban Girault, et al.
}

\section{- To cite this version:}

Aymeric Antoine-Lorquin, Ahmed Arnaoty, Sassan Asgari, Martine Batailler, Linda Beauclair, et al.. First landscape of binding to chromosomes for a domesticated mariner transposase in the human genome: diversity of genomic targets of SETMAR isoforms in two colorectal cell lines. 2017. hal01558001

\author{
HAL Id: hal-01558001 \\ https://hal.science/hal-01558001
}

Preprint submitted on 6 Jul 2017

HAL is a multi-disciplinary open access archive for the deposit and dissemination of scientific research documents, whether they are published or not. The documents may come from teaching and research institutions in France or abroad, or from public or private research centers.
L'archive ouverte pluridisciplinaire HAL, est destinée au dépôt et à la diffusion de documents scientifiques de niveau recherche, publiés ou non, émanant des établissements d'enseignement et de recherche français ou étrangers, des laboratoires publics ou privés. 


\section{First landscape of binding to chromosomes for a domesticated mariner transposase} in the human genome: diversity of genomic targets of SETMAR isoforms in two colorectal cell lines

Aymeric Antoine-Lorquin ${ }^{1,10}$, Ahmed Arnaoty ${ }^{2,10}$, Sassan Asgari ${ }^{3,10}$, Martine Batailler ${ }^{4,10}$, Linda Beauclair ${ }^{4,10}$, Catherine Belleanné $e^{1,10}$, Solenne Bire ${ }^{5,10}$, Nicolas Buisine ${ }^{6,10}$, Vincent Coustham $^{7,10}$, Alban Girault ${ }^{2,10}$, Serge Guyetant ${ }^{8,10}$, Thierry Lecomte ${ }^{2,10}$, Benoît Piégu ${ }^{4,10}$, Bruno Pitard ${ }^{9,10}$, Isabelle Stévant ${ }^{1,10}, \&$ Yves Bigot $^{4}$

${ }^{1}$ IRISA, 263 avenue du Général Leclerc, 35042 Rennes, France

${ }^{2}$ GICC, UMR CNRS 7292, 10 Boulevard Tonnellé, BP 3223, 37032 Tours, France

${ }^{3}$ School of Biological Sciences, The University of Queensland, Brisbane QLD 4072, Australia

${ }^{4}$ PRC, UMR INRA 0085 and CNRS 7247, Centre INRA Val de Loire, 37380 Nouzilly, France

${ }^{5}$ Institute of Biotechnology, Center for Biotechnology UNIL-EPFL, 1015 Lausanne, Switzerland

${ }^{6}$ UMR CNRS 7221, Muséum National d'Histoire Naturelle, Paris, France

${ }^{7}$ URA, UR INRA 0083, Centre INRA Val de Loire, 37380 Nouzilly, France

${ }^{8}$ Tumorothèque du CHRU de Tours, 37044 Tours cedex, France

${ }^{9}$ UMR INSERM U1087 and CNRS 6291, Nantes, France

${ }^{10}$ Author names were ranked in alphabetic order because all contributions were essential to the project managed by $\mathrm{Y}$. Bigot.

Corresponding author: yves.bigot@inra.fr 


\section{ABSTRACT}

Setmar is a 3-exons gene coding a SET domain fused to a Hsmar1 transposase. Its different transcripts theoretically encode 8 isoforms with SET moieties differently spliced. In vitro, the largest isoform binds specifically to Hsmar1 DNA ends and with no specificity to DNA when it is associated with hPso4. In colon cell lines, we found they bind specifically to two chromosomal targets depending probably on the isoform, Hsmar1 ends and sites with no conserved motifs. We also discovered that the isoforms profile was different between cell lines and patient tissues, suggesting the isoforms encoded by this gene in healthy cells and their functions are currently not investigated. 


\section{INTRODUCTION}

Setmar is one of the 52 neogenes identified in the human genome that originate from a DNA transposon [Supplementary Table S1], i.e. from the exaptation of an open reading frame (ORF) encoding a transposase, the enzyme is able to carry out all the DNA cleavage and strand transfer steps required for the transposition of this kind of transposable element (TE) [1]. Setmar appeared about 40-58 million years ago in the anthropoid lineage at the origin of the hominoids and the old and new world monkeys [2]. The gene is located on the human chromosome 3 (positions 4,292,212 to 4,328,658 in GRCh38/hg38) and arose from two neighboring genes, the first comprising two exons coding a lysine methyltransferase (SET) and the downstream second coding a Hsmar1 transposase (HSMAR1) belonging to the mariner family. Mariners are very simple TEs composed of one transposase ORF flanked by two inverted terminal repeats (ITRs). These flanking sequences are specifically bound by the transposase, that excises the TE from a 'donor' site before catalysing its insertion at another ('target') locus. During evolution of the anthropoid lineage, the accumulation of at least three mutations modified the expression properties of the SET and HSMAR1 genes. They acquired the ability to be transcribed in a single RNA transcript in which the SET domain can be N-terminally fused to the Hsmar1 moiety after intron excision to produce the SETMAR protein (also called Metnase) [2].

Recent work on Setmar RNA transcript variants isolated from the hematologic neoplasms of patients [3] and mRNA sequences available in databases revealed a complex situation. Alternative transcripts have the potential to code for two SET isoforms (V4 and X4) and eight SETMAR isoforms ranging from 40 to $78 \mathrm{kDa}$, although only the largest SETMAR isoform contains a complete SET moiety (Figure 1, Supplementary Table S2 and data S1). The eight SETMAR transcripts originate from alternative transcription start sites located upstream, within or downstream of the region encoding the SET domain, together with alternative splicing in the second exon of the SET moiety. Other variants resulting from single nucleotide polymorphisms can be found in human populations ([3] and http://databases.lovd.nl/whole_genome/view/SETMAR). The detection of SETMAR in various colorectal [4], melanoma, breast cancer (Supplementary Figure S1), and leukaemia cell lines [5] confirmed that only five transcript variants were translated into protein isoforms (Figure 1, V1, V2, X2, V5 and an isoform with a molecular weight (MW) similar to that of HSMAR1), although differences exists from one cell line to another [4]. To our knowledge, it is not established which protein isoforms are present in healthy and cancerous tissues of patients. 
Since a decade, significant efforts have been carried out to elucidate the enzymatic activities of the SET and HSMAR1 moieties, to identify cellular protein partners, and to integrate this knowledge in biological pathways. In summary, the SET moiety was associated with the dimethylation of histone 3 lysines 4 and 36 [6] and the methylation of lysine 130 of the splicing factor snRNP70 [7]. The N-terminal domain of the HSMAR1 moiety has kept its ability to bind specifically to the ITRs of the Hsmar1 transposon in vitro. Its C-terminal domain displays most of the cleavage and strand transfer activities of mariner transposases, except that the $3^{\prime}$ second strand cleavage at the outer ITR ends is severely affected by a mutation of the catalytic triad (DDN instead of DDD) [8,9]. SETMAR has an additional DNA binding activity, in the form of a stable complex with the hPso4 protein $[10,11]$, that is able to bind in vitro to non-ITR double-stranded DNA target. To date, it is not clear whether the different SETMAR isoforms can bind to chromosomal DNA in human cells and whether they target specific Hsmar1 ITR related sequences and-or nonITRs. From a functional standpoint, the biological activities of the SETMAR V1 domains (Figure 1) have been documented in the context of at least three housekeeping mechanisms, where the protein enhances chromosomal decatenation [12,13], improves the efficiency and the accuracy of DNA repair by non-homologous end-joining [14-17], and positively impacts the restart of stalled replication forks after DNA damage repair [18]. Interestingly, SETMAR V1 is also able to enhance the chromosomal integration of transfected DNA fragments [19] and of lentiviral DNA into host cells genome [20]. However, the precise molecular mechanism is unknown and whether the choice of integration sites is governed by the binding properties of SETMAR is still an open question. Also, it is not clear whether (and how) this mechanism is connected to metastatic processes, by mediating integration of the free DNA released by tumor cells into the chromosomal DNA of healthy cells $[21,22]$.

Here, we derived the DNA binding landscape of several SETMAR isoforms in the colorectal cell lines SW48 and HT29. These lines display specific expression profiles of SETMAR isoforms: HT29 cells express a single SETMAR isoform (V2) whereas SW48 cells express several (V1, V2, X2, V5 and HSMAR1) [4]. We also revisit the annotation of the human genome for the Hsmar1 element and its associated 80-pb miniature element, MADE1, using logol [23] and BLAST+, and we could detect and annotate $\sim 2500$ novel MADE1 copies. We found that SETMAR binding sites occur mostly at MADE1 sequences, although a significant fraction of them (up to $~ 50 \%$ ) can also be found at unannotated regions. In addition, we document the expression profile of SETMAR isoforms in healthy and cancerous colorectal tissues. Strikingly, X2 and V2 are the only SETMAR isoforms 
expressed in both healthy and cancerous colon biopsies. We discuss which SETMAR isoforms are found in healthy tissues and tumors, with respect to the interest to study this protein in cancerous lines.

\section{MATERIALS AND METHODS}

\section{Culture of cell lines}

HeLa cells, human colorectal cancer cell lineages (SW48, SW403, HT29), human melanoma cell lineages (BRIS, SK-MEL28and 518-A2), and human breast cancer cell lineages (MCF7, MDA-MB231, SKBR3 and T47D) were all cultured in Dulbecco's modified Eagle's medium (DMEM) supplemented with $10 \%$ fetal bovine serum (FBS), at $37^{\circ} \mathrm{C}$ and with $5 \% \mathrm{CO}_{2}$. HeLa cell transfection with $1 \mu \mathrm{g}$ of pVAX-HSMAR1 DNA was monitored as described [4].

\section{Samples of non-tumoral and tumoral colon tissues}

Two samples of colon tissues, tumoral and adjacent non-tumoral tissues, were recovered from patients after surgery for a colorectal cancer in 2007 or 2008 . Samples were stored at $-80^{\circ} \mathrm{C}$ by the tumor bank of the Tours CHRU. Patients were informed of the possibility of the use of levies for research and their agreement to participate in this research was collected. Selected samples did not display more than $50 \%$ of tumoral cells (Supplementary Table S3).

\section{Protein extraction from non-tumoral and tumoral colon tissues}

Tissue slices $(10 \mu \mathrm{m})$ were generated with a cryomicrotome on frost samples of tumoral and non-tumoral tissues, and stored at $-80^{\circ} \mathrm{C}$. For each patient, protein lysates were made by suspending a tenth of slices in iced RIPA buffer $(20 \mathrm{mM}$ Tris- $\mathrm{HCl}$ pH7.2, $150 \mathrm{mM} \mathrm{NaCl}$, $1 \mathrm{mM}$ EDTA, 1\% Glycerol, 1\% Triton X100, 0.5\% doxycholate, 0.1\% SDS, 1X-Complete Protease Inhibitor Cocktail (Roche Applied Sciences, Meylan, France)), vortexed for 1 min, incubated on ice for $15 \mathrm{~min}$, centrifuged at $15,000 \mathrm{~g}$ for $15 \mathrm{~min}$ at $4^{\circ} \mathrm{C}$. After recovery of supernatants, proteins were quantified with a Quick Start ${ }^{\mathrm{TM}}$ Bradford Protein Assay (BioRad, Richmond, USA) and conserved at $-20^{\circ} \mathrm{C}$.

\section{Antibody purification}


Murine pre-immune and anti-HSMAR1 polyclonal sera were produced by DNA vaccination using ICANtibodies ${ }^{\mathrm{TM}}$ technology (In Cell Art, Nantes, France) as described [4]. Polyclonal antibodies $(\mathrm{pA})$ contained in pre-immune and anti-HSMAR1 murine sera were purified using Protein A/G MagBeads (GenScript, Piscataway, USA). Their quality was then verified by polyacrylamide gel electrophoresis (PAGE) after staining with Coomassie blue and their concentration was defined using the BCA Protein quantification Kit (Interchim, Montluçon, France).

\section{PAGE, immunoblotting, and hybridization of antisera}

Protein extracts from cultured cells, PAGE, transfer onto a polyvinylidene difluoride (PVDF) membrane, antibodies incubations and imaging with a FUJI LAS4000 imager were carried out as described [4].

\section{ChIP experiments}

Chromatin samples were prepared from non-synchronous and exponentially growing SW48 and HT29 cells. Chromatin shearing was performed with a Bioruptor ultrasonicator (Diagenode, Belgium). Chromatin immunoprecipitation was performed with $10 \mu \mathrm{g}$ of purified pre-immune or HSMAR1 pA, and purification of immunoprecipitated DNA was done using the iDeal ChIP-Seq kit following supplier's recommendations (Diagenode, Ougrée, Belgium). Libraries for Illumina sequencing were made using iDeal ChIP-Seq \& Library Preparation Kit (Diagenode, Ougrée, Belgium). DNA quantities were monitored at various steps of the procedure with the Qubit尺 dsDNA HS Assay Kit (Molecular probes, Eugene, USA). Fragment size selection, library quality control and Illumina sequencing (HiSeq 51 nucleotides, TruSeq SBS Kit v3 (Illumina, Fulbourn, United Kingdom)) were achieved at the Imagif sequencing platform (CNRS, Gif-sur-Yvette, France), following published quality recommendations [24]. Data published in this paper are based on three biological replicates.

\section{ChIP-Seq Analyses}

ChIP-Seq sequence reads were mapped to the human genome assembly hg38 (December 2013; available at http://www.ncbi.nlm.nih.gov/assembly/GCF_000001405.26) with the Bowtie short read aligner [25]. Peak calling was done with the peak-calling prioritization pipeline (PePr1.1.5) [26] from bam files, and normalized over input (three 
biological replicates).

\section{Data analyses}

Annotation of Hsmar1 and MADE1 copies in hg38 is described in Supplementary data 2. Unique or intersecting annotations were computed using bedtools. Ontologies and search for conserved motifs were carried out with GLAM2 [27], the GREAT pipeline [28], and the RSAT pipeline [29,30]. Data analyses and most graphic representation were done with $\mathrm{R}$ (https://www.r-project.org/). Graphic representations of chromosomes in the hg38 genome model were performed using svg files calculated with the DensityMap software at http://chicken-repeats.inra.fr/launchDM_form.php and gff files parameterized as recommended [31] in order to supply the chromosomes sizes, the locations of centromers, and a suitable description of the third field, i.e. the features in column 3 . The locations of each centromer in chromosomes of the hg19 model were recovered and updated with liftover at the UCSC web site.

\section{Data repository}

All raw and processed data are available through the European Nucleotide Archive under accession number PRJEB19196. The gff files describing the updated annotation of Hsmar1 and MADE1 copies in the hg38 release and the ChIP-Seq peaks were supplied as Supplementary data 3 and 4.

\section{RESULTS}

\section{SETMAR binding sites along human chromosomes}

Chromosomal targets of SETMAR were identified by ChIP-Seq on cell lines HT29 and SW48, using a custom made polyclonal antibody directed against the HSMAR1 domain of SETMAR. The specificity of this key antibody has been addressed previously which showed a good specificity/sensitivity ratio [4]. Our experience is that none of the commercial monoclonal and polyclonal antibodies commercially available and directed against the HSMAR1 domain are efficient are for ChIP experiments. ChIP-Seq of panHSMAR1/SETMAR was preferred to transient transfection of plasmids expressing individual SETMAR isoforms because transfected cell populations typically display heterogeneous expression levels that can span three orders of magnitude. This may not 
reflect the expression levels found in vivo [33] and can have strong impact on the binding landscape. Indeed, HSMAR1 and SET were found to accumulate at nucleoli (or periheterochromatin) when overexpressed in HeLa [33] and other cell lines (http://www.proteinatlas.org/ENSG00000170364-SETMAR/subcellular). Overall, we found 4366 and 8425 binding sites (BS) in HT29 and SW48 cells, respectively. Taking into account that in a few cases, peaks found in one cell type were split into two in the other, there is a total of 3513 peaks in common, together with 796 and 4908 only found in HT29 or SW48 cells.

Unfortunately, defining the co-occurrence of SETMAR BS with Hsmar1 and MADE1 copies proved to be difficult, mainly because of their limited description in hg38 genome annotation. TEs annotation is notoriously difficult to carry out and it is not uncommon to assess its quality and re-annotate a particular family/subfamily. The quality of the RepeatMasker annotation was therefore verified and improved using logol and BLAST+ (Supplementary data 2). We produced a revised annotation describing 519 Hsmar1 copies (displaying to 615 ITRs), together with 10295 MADE1 copies composed of 1875 full-length elements, 6246 elements truncated at one end (displaying a single ITR), and 2174 elements with damaged ITRs (outer ends lacking $\geq 5$ nucleotides).

Intersections between ChIP-Seq peaks and the Hsmar1 or MADE1 copies were calculated with bedtools using default parameters. Overall, there are $3549(\sim 83.9 \%)$ and 3998 $(\sim 49.6 \%)$ BS at Hsmar1 or MADE1 copies in HT29 and SW48 cells, respectively, the vast majority of which $(78.5 \%, 3449 / 4396)$ is common to both cell lines. Binding occurs mostly at MADE1 elements compared to Hsmar1 ITRs, in both cell lines (Table 1). This result is consistent with the fact that SETMAR V2 is expressed in both cell lines (and likely other isoforms as well, in SW48 cells) and can bind to MADE1 and Hsmar1 DNA in vivo. A total of 456 Hsmar1 and 6062 MADE1 copies are not bound by SETMAR. We thus asked whether these copies were associated with lamina (LAD) or nucleolus (NAD) associated domains ([34] and http://biorxiv.org/content/early/2016/05/24/054908), but we could find no statistical correlation. Similarly, we could not find any association with intergenic regions, genes body, $5^{\prime}$ proximal and $3^{\prime}$ distal region of genes and introns.

We next characterized the BS common to both cell lines and overlapping with truncated MADE1 copies. Since they are composed of a single ITR, they minimize the confounding effect of nearby peaks. BS are centered on a sequence corresponding to positions 5 to 26 of Hsmar1 and MADE1 ITRs (Figure 2a, Supplementary data 2). We note, however, that while residues located at position 8 to 17 and 21 to 26 are critical for HSMAR1 binding in 
vitro [2, 35], the conserved CG dinucleotide located at positions 24 and 25 appears less common at the genomic DNA level. On average, BS display $92.8 \pm 3.8 \%$ identical residues with the MADE1 consensus. In contrast, we found more sequence diversity in unbound MADE1 sequences, with an average of $87.8 \pm 5.2 \%$ identical residues.

Thus, our results support well the fact that SETMAR V2 (and likely of other isoforms) binding to MADE1 and Hsmar1 DNA depends on the sequence conservation of their ITR, but it also depends on cell specific host factor(s).

\section{Features of non-ITR binding targets along human chromosomes}

The number of BS outside of Hsmar1 or MADE1 copies was higher in SW48 than in HT29 cells, which correlated with the presence of four other SETMAR isoforms in SW48 (V1, X2, V5 and HSMAR1) that were dramatically more abundant than or absent in HT29 cells. On average, these BS are associated to lower q-values and smaller widths than those found at Hsmar1 or MADE1 DNA BS in HT29 and SW48 cells (Supplementary Figure S2; Figure 2b). Altogether, this suggests that they require a specific molecular machinery, which may be independent of the presence of hPSO4 in both cell lines, as this protein is present in similar amounts in both cell lines (Figure 3).

Searches for common ontologies and conserved transcription factor (TF) BS were conducted using the GREAT pipeline with default parameters (Supplementary data 5). We could not find significant association between BS at Hsmar1/MADE1 sequences, gene function or their location with respect to genes. For BS located outside of Hsmar1/MADE1 sequences, 200 of the 4783 these BS could be associated to genes involved in epithelia and mesenchymal stem cell biology.

As expected in term of genomic distribution, BS localized at ITRs followed that of Hsmar1MADE1 copies. Unexpectedly, however, more than $75 \%$ of the BS located outside of Hsmar1/MADE1 sequences were not distributed at random and were clustered in a limited number of regions, ranging from 1 to $20 \mathrm{Mbp}$, found in only 10 chromosomes (Figure 4). Although no common DNA motif could be found, BS characteristics and their genomic distribution along chromosomes certainly reflect the (direct or indirect) specific binding of some SETMAR isoforms to DNA or chromatin. We next searched for putative transcription factor binding motifs (TFBM) at BS located outside of Hsmar1/MADE1, with the RSAT pipeline (Supplementary data 5). We found a clear signature of EGR1 and-or MEF2 TFBM in $69 \%$ of them, suggesting possible physical interactions between the two 
TFs and SETMAR isoforms.

\section{Expression of SETMAR isoforms in colon biopsies}

Due to the heterogeneity of SETMAR isoform profiles between cancerous cell lines $([4,13]$ and Supplementary Figure S1) and that the expression profiles of healthy tissues or cancerous tumors can drift in primary cultures as well as in established cell lines [36-38], the presence of SETMAR isoforms was probed in biopsies of non-tumoral and tumoral colon tissues from 26 patients affected by a colon cancer. As a control, we also used a colorectal biopsy from a patient with no colorectal disorder. In healthy and tumoral colon tissues, we found that four (V1, V2, V5 and HSMAR1) of the five isoforms present in HT29 and SW48 cells were not detected but two other SETMAR isoforms, X2 and V3, were present (Figure 5a). The most abundant was the X2 isoform. Its expression level varies from 1 to 5 -folds, depending on the nature of the biopsy, and the patient but not sex (Figure 5b). This isoform is also found in healthy tissues. The second protein, which was poorly expressed only in a few patients, has a molecular weight of $55 \mathrm{kDa}$ that corresponds to the V3 isoform. Overall, the stark contrast between the SETMAR expression profile in vivo (healthy/cancerous tissues) and in vitro (established colorectal cell lines) shows that it is very sensitive to the cellular context. Interestingly, V2, X2 and V3 isoforms likely have altered or no SET catalytic activity since important parts of this domain are missing in these isoforms. Thus, the resulting proteins would be equivalent to a HSMAR1 tagged with N-terminal peptides that might modulate their interactions with putative (chromatin) partners.

\section{DISCUSSION}

Our work shows for the first time that SETMAR binds at a variety of BS in vivo. As expected, binding occurs at the ITR of Hsmar1 and MADE1, but also occurs at other sites centered on GC-rich regions with no obvious conserved motif. Remarkably, in HT29 cells, V2 binding occurs despite abundant hPSO4 levels in the nucleus. Overall, this novel population of BS unlikely result from the direct binding of SETMAR to chromosomal DNA, but may be mediated by other protein partners, possibly EGR1 and/or MEF2, that would interact through the pre-SET and/or the HSMAR1 domain.

The V2 isoform lacks intact SET and post-SET domains, which rules out indirect binding mediated by protein complexes involved in replication or DNA repair $[3,5,18]$. They would 
therefore result from interactions with DNA binding proteins that were so far not described and which would occur with the pre-SET subdomain and/or the HSMAR1 domain. In SW48, because the X2 and V5 isoforms and HSMAR1 display no SET subdomains, it is likely that these proteins have interactions with chromosomal DNA that are similar to those of V2. Under this hypothesis, most of the peaks unrelated to Hsmar1 and MADE1 copies would therefore result from interactions between the V1 isoform and other protein partners, putatively EGR1 and MEF2. These peaks would likely not result from interactions between V1 and protein complexes involved in replication or DNA repair. Indeed, they are discrete and well defined, what is not expected in such interactions that are not specific in location in non-synchronized cell populations.

Our work, although limited to a cohort of 26 human samples, already provides important new data. Remarkably, our results demonstrate that the study of SETMAR functions using cell lines is tricky, especially for the V1 isoform that was so far never described as being present in healthy organs or in tumors in vivo. Here, our study was limited to 26 patients in whom only the V3 and X2 isoforms were detected in non-tumoral and certain cancerous biopsies. Currently, we have analysed 20 cell lines derived from colorectal cancers (13; [4] and Figure 2A, lane 1), melanoma (3) and breast cancers (4) (Supplementary Figure S1). In none of them the V3 isoform was detected while the V5 isoform was found in 7 colorectal lines. The V2 and V3 isoforms display both a damaged SET domain likely unable to bind to DNA. It is therefore reasonable to hypothesize that the ability of V3 to bind to chromosomal DNA is close to that of V2 and mainly occurred on ITR of Hsmar1 and MADE1 copies. Currently, it cannot be discarded that most non-Hsmar1/MADE1 BS are due to the binding of $\mathrm{V} 1$, a SETMAR isoform that was so far only detected in a few cell lineages.

Since HSMAR1 3' end cleavage activity is severely impaired [8], HSMAR1 and isoforms like V2, V3, X2 and V5 are unlikely to mediate Hsmar1MADE1 excision. However, HSMAR1 is still capable of mediating $5^{\prime}$ end cleavage and DNA integration $[8,19]$. This raises the interesting possibility that SETMAR isoforms might catalyse integration of extracellular DNA, because circulating free DNA is often released from cell death or infection and can be passively transferred into cells [21,22]. Given the limited number of possible integration sites, mostly located outside of genes, SETMAR would then represent a nuclear mechanism protecting cells against the genotoxic effects of integration of circulating cell free DNA. This would represent a recent functional innovation, restricted to the anthropoid lineage. 


\section{FUNDINGS}

This work was funded by the C.N.R.S., the I.N.R.A., and the GDR CNRS 2157. It also received funding from a Research Program grant from the Cancéropôle Grand-Ouest, the Ligue Nationale Contre le Cancer and grants from Amgen and the French National Society of Gastroenterology. 


\section{REFERENCES}

1. Piégu,B., Bire,S., Arensburger,P. and Bigot,Y. (2015) A survey of transposable element classification systems--a call for a fundamental update to meet the challenge of their diversity and complexity. Mol Phylogenet Evol., 86, 90-109.

2. Cordaux,R., Udit,S., Batzer,M.A. and Feschotte,C. (2006) Birth of a chimeric primate gene by capture of the transposase gene from a mobile element. Proc Natl Acad Sci USA., 103, 8101-8106.

3. Jeyaratnam,D.C., Baduin,B.S., Hansen,M.C., Hansen,M., Jørgensen,J.M., Aggerholm,A., Ommen,H.B., Hokland,P. and Nyvold,C.G. (2014) Delineation of known and new transcript variants of the SETMAR (Metnase) gene and the expression profile in hematologic neoplasms. Exp Hematol., 42, 448-456.

4. Arnaoty,A., Gouilleux-Gruart,V., Casteret,S., Pitard,B., Bigot,Y. and Lecomte,T. (2013) Reliability of the nanopheres-DNA immunization technology to produce polyclonal antibodies directed against human neogenic proteins. Mol Genet Genomics., 288, 347363.

5. Wray,J., Williamson,E.A., Chester,S., Farrington,J., Sterk,R., Weinstock,D.M., Jasin,M., Lee,S.H., Nickoloff,J.A. and Hromas,R. (2010) The transposase domain protein Metnase/SETMAR suppresses chromosomal translocations. Cancer Genet Cytogenet., 200, 184-190.

6. Fnu,S., Williamson,E.A., De Haro,L.P., Brenneman,M., Wray,J., Shaheen,M., Radhakrishnan,K., Lee,S.H., Nickoloff,J.A. and Hromas,R. (2011) Methylation of histone $\mathrm{H} 3$ lysine 36 enhances DNA repair by nonhomologous end-joining. Proc Natl Acad Sci USA., 108, 540-545.

7. Carlson,S.M., Moore,K.E., Sankaran,S.M., Reynoird,N., Elias,J.E. and Gozani,O. (2015) A proteomic strategy identifies lysine methylation of splicing factor snRNP70 by the SETMAR Enzyme. J Biol Chem., 290, 12040-12047.

8. Liu,D., Bischerour,J., Siddique,A., Buisine,N., Bigot,Y. and Chalmers,R. (2007) The human SETMAR protein preserves most of the activities of the ancestral Hsmar1 transposase. Mol Cell Biol., 27, 1125-1132.

9. Miskey,C., Papp,B., Mátés,L., Sinzelle,L., Keller,H., Izsvák,Z. and Ivics,Z. (2007) The ancient mariner sails again: transposition of the human Hsmar1 element by a reconstructed transposase and activities of the SETMAR protein on transposon ends. Mol Cell Biol., 27, 4589-4600.

10. Beck,B.D., Park,S.J., Lee,Y.J., Roman,Y., Hromas,R.A. and Lee,S.H. (2008) Human Pso4 is a metnase (SETMAR)-binding partner that regulates metnase function in DNA 
repair. J Biol Chem., 283, 9023-9030.

11. Beck,B.D., Lee,S.S., Hromas,R. and Lee,S.H. (2010) Regulation of Metnase's TIR binding activity by its binding partner, Pso4. Arch Biochem Biophys., 498, 89-94.

12. Williamson,E.A., Rasila,K.K., Corwin,L.K., Wray,J., Beck,B.D., Severns,V., Mobarak,C., Lee,S.H., Nickoloff,J.A. and Hromas,R. (2008) The SET and transposase domain protein Metnase enhances chromosome decatenation: regulation by automethylation. Nucleic Acids Res., 36, 5822-5831.

13. Wray,J., Williamson,E.A., Sheema,S., Lee,S.H., Libby,E., Willman,C.L., Nickoloff,J.A. and Hromas,R. (2009) Metnase mediates chromosome decatenation in acute leukemia cells. Blood., 114, 1852-1858.

14. Hromas,R., Wray,J., Lee,S.H., Martinez,L., Farrington,J., Corwin,L.K., Ramsey,H., and Nickoloff,J.A. and Williamson,E.A. (2008) The human set and transposase domain protein Metnase interacts with DNA Ligase IV and enhances the efficiency and accuracy of non-homologous end-joining. DNA Repair., 7,1927-1937.

15. Wray,J., Williamson,E.A., Chester,S., Farrington,J., Sterk,R., Weinstock,D.M., Jasin,M., Lee,S.H., Nickoloff,J.A. and Hromas,R. (2010) The transposase domain protein Metnase/SETMAR suppresses chromosomal translocations. Cancer Genet Cytogenet., 200, 184-90.

16. Beck,B.D., Lee,S.S., Williamson,E. and Hromas,R.A., Lee,S.H. (2011) Biochemical characterization of metnase's endonuclease activity and its role in NHEJ repair. Biochemistry., 50, 4360-4370.

17. Kim,H.S., Chen,Q., Kim,S.K., Nickoloff,J.A., Hromas,R., Georgiadis,M.M. and Lee,S.H. (2014) The DDN catalytic motif is required for Metnase functions in non-homologous end joining (NHEJ) repair and replication restart. J Biol Chem. 289, 10930-10938.

18. Kim,H.S., Kim,S.K., Hromas,R. and Lee,S.H. (2015) The SET Domain Is Essential for Metnase Functions in Replication Restart and the 5' End of SS-Overhang Cleavage. PLoS One., 10, e0139418.

19. Lee,S.H., Oshige,M., Durant,S.T., Rasila,K.K., Williamson,E.A., Ramsey,H., Kwan,L., Nickoloff,J.A. and Hromas,R. (2005) The SET domain protein Metnase mediates foreign DNA integration and links integration to nonhomologous end-joining repair. Proc Natl Acad Sci USA., 102, 18075-18080.

20. Williamson,E.A., Farrington,J., Martinez,L., Ness,S., O'Rourke,J., Lee,S.H., Nickoloff,J. and Hromas,R. (2008) Expression levels of the human DNA repair protein metnase influence lentiviral genomic integration. Biochimie., 90, 1422-1426.

21. García-Olmo,D.C., Domínguez,C., García-Arranz,M., Anker,P., Stroun,M., García- 
Verdugo,J.M. and García-Olmo,D. (2010) Cell-free nucleic acids circulating in the plasma of colorectal cancer patients induce the oncogenic transformation of susceptible cultured cells. Cancer Res., 70, 560-567.

22. Füri,I., Kalmár,A., Wichmann,B., Spisák,S., Schöller,A., Barták,B., Tulassay,Z. and Molnár,B. (2015) Cell Free DNA of Tumor Origin Induces a 'Metastatic' Expression Profile in HT-29 Cancer Cell Line. PLoS One., 10, e0131699.

23. Belleannée,C., Sallou,O. and Nicolas,J. (2014) Logol: Expressive Pattern Matching in sequences. Application to Ribosomal Frameshift Modeling. In Comin,M., Kall,L., Marchiori,E., Ngom, A., Rajapakse,J. (eds.), PRIB2014 - Pattern Recognition in Bioinformatics, 9th IAPR International Conference, Aug 2014. Springer International Publishing, Stockholm, Vol. 8626, pp.34-47.

24. Bailey,T., Krajewski,P., Ladunga,I., Lefebvre,C.,Li,Q., Liu,T., Madrigal,P., Taslim,C. and Zhang,J. (2013) Practical guidelines for the comprehensive analysis of ChIP-seq data. PLoS Comput Biol., 9, e1003326.

25. Langmead,B. and Salzberg,S.L. (2012) Fast gapped-read alignment with Bowtie 2. Nat Methods., 9, 357-359.

26. Zhang,Y., Lin,Y.H., Johnson,T.D., Rozek,L.S. and Sartor,M.A. (2014) PePr: a peakcalling prioritization pipeline to identify consistent or differential peaks from replicated ChIP-Seq data. Bioinformatics., 30, 2568-2575.

27. Frith,M.C., Saunders,N.F.W., Kobe,B. and Bailey,T.L. (2008) Discovering sequence motifs with arbitrary insertions and deletions, PLoS Comp Biol., 4, e1000071.

28. McLean,C.Y., Bristor,D., Hiller,M., Clarke,S.L., Schaar,B.T., Lowe,C.B., Wenger,A.M. and Bejerano,G. (2010) GREAT improves functional interpretation of cis-regulatory regions. Nat. Biotechnol., 28, 495-501.

29. Thomas-Chollier,M., Herrmann,C., Defrance,M., Sand,O., Thieffry,D. and van Helden,J. (2011). RSAT peak-motifs: motif analysis in full-size ChIP-seq datasets Nucleic Acids Res., 40:e31.

30. Thomas-Chollier,M., Darbo,E., Herrmann,C., Defrance,M., Thieffry,D. and van Helden,J. (2012). A complete workflow for the analysis of full-size ChIP-seq (and similar) data sets using peak-motifs. Nat Protoc., 7:1551-1568.

31. Guizard,S., Piégu,B. and Bigot,Y. (2016) DensityMap: a genome viewer for illustrating the densities of features. BMC Bioinformatics., 17, 204.

32. Demattei,M.V., Hedhili,S., Sinzelle,L., Bressac,C., Casteret,S., Moiré,N., Cambefort,J., Thomas,X., Pollet,N., Gantet,P., et al. (2011) Nuclear importation of Mariner transposases among eukaryotes: motif requirements and homo-protein interactions. 
PLoS One., 6, e23693.

33. Bire,S., Casteret,S., Arnaoty,A., Piégu,B., Lecomte,T. and Bigot,Y. (2013) Transposase concentration controls transposition activity: myth or reality? Gene., 530, 165-171.

34. Kind,J., Pagie,L., de Vries,S.S., Nahidiazar,L., Dey,S.S., Bienko,M., Zhan,Y., Lajoie,B., de Graaf,C.A., Amendola,M., et al. (2015) Genome-wide maps of nuclear lamina interactions in single human cells. Cell., 163, 134-147.

35. Roman,Y., Oshige,M., Lee,Y.J., Goodwin,K., Georgiadis,M.M., Hromas,R.A. and Lee,S.H. (2007) Biochemical characterization of a SET and transposase fusion protein, Metnase: its DNA binding and DNA cleavage activity. Biochemistry., 46, 11369-11376.

36. Havelock,J.C., Rainey,W.E. and Carr,B.R. (2004) Ovarian granulosa cell lines. Mol Cell Endocrinol., 228, 67-78.

37. Song,X.C., Fu,G., Yang,X., Jiang,Z., Wang,Y. and Zhou,G.W. (2008) Protein expression profiling of breast cancer cells by dissociable antibody microarray (DAMA) staining. Mol Cell Proteomics., 7, 163-169.

38. Pan, C.,Kumar,C., Bohl,S., Klingmueller,U. and Mann,M. (2009) Comparative proteomic phenotyping of cell lines and primary cells to assess preservation of cell typespecific functions. Mol Cell Proteomics., 8, 443-540. 


\section{Figure Legends}

Figure 1. Organisation of the Setmar gene (a) and the various isoforms resulting from its transcription (b).

Figure 2. Graphic analyses of ChIP-Seq peak features. (a) Conserved motifs located by GLAM2 in truncated MADE1 copies overlapping or not with a ChIP-Seq peak. On the top, the SETMAR BS sequence in the MADE1 consensus sequence is shown, and below the sequence of the BS defined in vitro [2,35]. Positions along the ITR are indicated below the horizontal axes of Weblogos. The population of MADE1 without peaks was heterogeneous BS and since it is a mixture of elements with few conserved BS and elements with an ability to be bound by SETMAR isoforms that depends on the local chromatin structure. (b) Boxplots representing the distributions of the width (in bp) of peaks colocalizing with Hsmar1 or MADE1 ITRs (2 boxes on the right) or elsewhere in the human genome (2 boxes on the left) depending on the cell lines (HT29 in blue, SW48 in red).

Figure 3. Control of the hPso4 (also known as PRPF19) presence in HT29 and SW48 cell lines. Protein extracts $(50 \mu \mathrm{g})$ were used to make Western blot. hPso4 and beta actin detections were achieved together using a rabbit anti-human hPso4 (PA5-24797, ThermoFischer Scientific) and a rabbit anti-human beta actin (EPR16769, Abcam) as primary antibodies and donkey anti-rabbit IgG-IR DYE800LT as a secondary antibody. Incubation with both antibodies were done overnight at $4^{\circ} \mathrm{C}$ in the Odyssey blocking buffer TBS (LI-COR). Three washings (10 min) of membranes were done in PBS1X, 0.1\% triton $\mathrm{X} 100$, at room temperature after each antibody incubation. Membranes were analysed using an Odyssey CLx Imaging system (LI-COR). Protein molecular weights are indicated in the left margin.

Figure 4. Distribution of gene densities, Hsmar1 and MADE1 copies and peaks in chromosomes of the hg19 genome model. (a) Graphic representation of the gene density, and the occurrences of MADE1 and Hsmar1 copies and ChIP-Seq peaks colocalized with a Hsmar1 or a MADE1 copy along the human chromosomes. Each chromosome is represented from the left to the right in six columns: the gene density, the occurrences of MADE1 copies, the occurrences of Hsmar1 copies, the occurrences of ChIP-Seq peaks co-localized with a Hsmar1 or a MADE1 copy only in HT29 cells, both in 
HT29 and SW48 cells, and only in SW48 cells. (b) Graphic representation of the gene density, and the occurrences of MADE1 and Hsmar1 copies and the ChIP-Seq peaks that were not co-localized with a Hsmar1 or a MADE1 copy along the human chromosomes. Each chromosome is represented from the left to the right in five columns: the gene density, the occurrences of MADE1 copies, the occurrences of Hsmar1 copies, the occurrences of ChIP-Seq peaks that did not co-localize with a Hsmar1 or a MADE1 copy only in HT29 cells, and only in SW48 cells. The most concentrated region of non-ITR binding targets in HT29 and SW48 is indicated with a light green bar. In both graphics, gene density and ChIP-Seq peaks were colourized using the colour scale 1, the occurrences of MADE1 and Hsmar1 copies used the colour scale 2. Densities and occurrences were calculated per window of $10^{5} \mathrm{bp}$. The centromer of each chromosome is located by a constriction in the shape of each chromosome.

Figure 5. Western blot analyses of SETMAR isoforms in healthy and tumorous colorectal tissues of patients. (a) Protein extracts of two biopsies from patient 12 (P12; healthy $(H)$ and tumoral $(\mathrm{T})$ tissues, $50 \mu \mathrm{g}$ ), SW403 cell line that mainly expresses V2 and X2 SETMAR isoforms [4] (lane 1,5 $\mathrm{g}$ ), HeLa cells (lane 2, $50 \mu \mathrm{g}$ ), HeLa cells transfected with pVAX-Hsmar1 (lane 3, $50 \mu \mathrm{g}$ ), and a colorectal biopsy from a patient with no colorectal disorder (lane 4,50 $\mu \mathrm{g}$ ). Molecular weights of isoforms are indicated in the left margin. (b) Protein extracts from healthy $(H)$ and tumoral $(T)$ tissue biopsies of 26 patients ( $P 1$ to P26). Actin is shown as an internal loading reference. The sex of each patient and the phenotype of their colon cancer (microsatellite stable (MSS) and microsatellite instable (MSI)) are indicated. For imaging the Western blot results with the anti-Hsmar1 antibodies in (a) and (b), an exposure time of 1 hour was used while only 5 to 10 minutes were required for the actin controls (Abcam, ab13822). Protein molecular weights are indicated in the left margin. 
Table 1a. Number and location of ChIP-Seq peaks in two colorectal cell lines

\begin{tabular}{|c|c|c|c|}
\hline Binding sites & HT29 cells & SW48 cells & $\begin{array}{c}\text { Common in both cell } \\
\text { lines }\end{array}$ \\
\hline Hsmar1 & 19 & 21 & 123 \\
\hline MADE1 & 197 & 710 & 3326 \\
\hline Other & 538 & 4082 & 163 \\
\hline
\end{tabular}

Table 1b. Proportion of Hsmar1 and MADE1 ITRs bound by SETMAR in two colorectal cell lines

\begin{tabular}{|l|c|c|}
\hline & $\mathrm{HT} 29$ & $\mathrm{SW} 48$ \\
\hline $\begin{array}{l}\text { Proportion of Hsmar1 ITRs } \\
\text { bound in hg38 }\end{array}$ & $23.2 \%$ & $23.6 \%$ \\
\hline $\begin{array}{l}\text { Proportion of MADE1 ITRs } \\
\text { bound in hg38 }\end{array}$ & $34.2 \%$ & $39.7 \%$ \\
\hline
\end{tabular}


a. Setmar exon-intron organisation

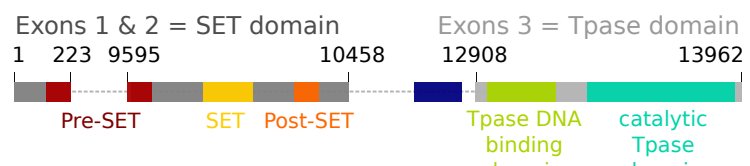

b. Domain organisation of SETMAR isoforms

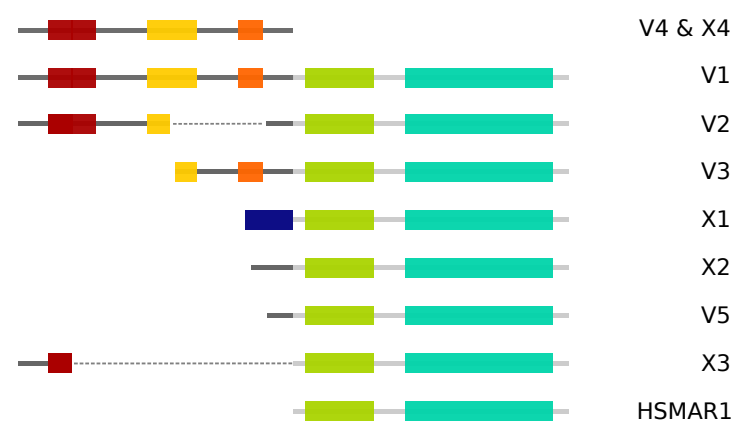


a. MADE1 BS: GGTGCAAAAGTAATTGCGG Functional BS: GGTGCAAAAGNNNTTGCGG

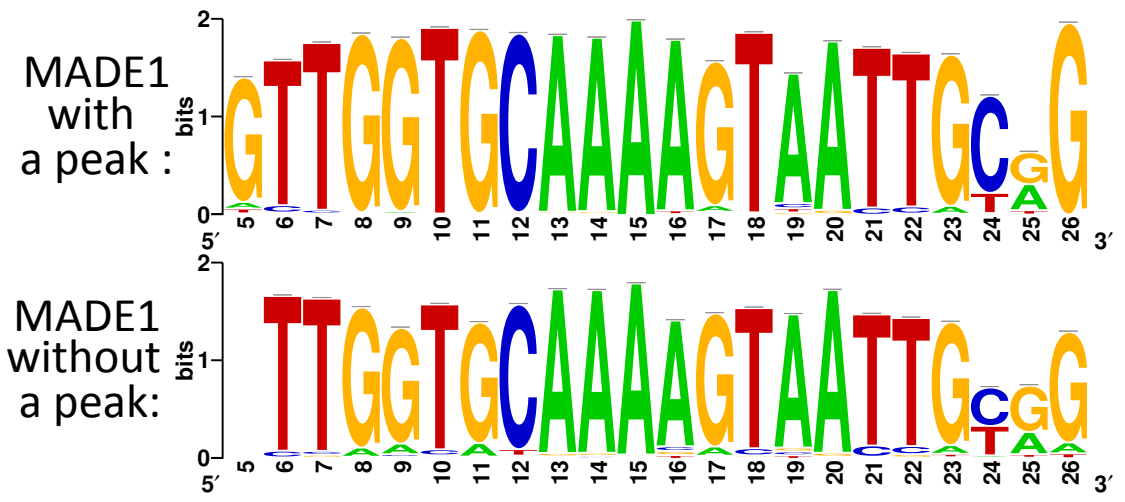

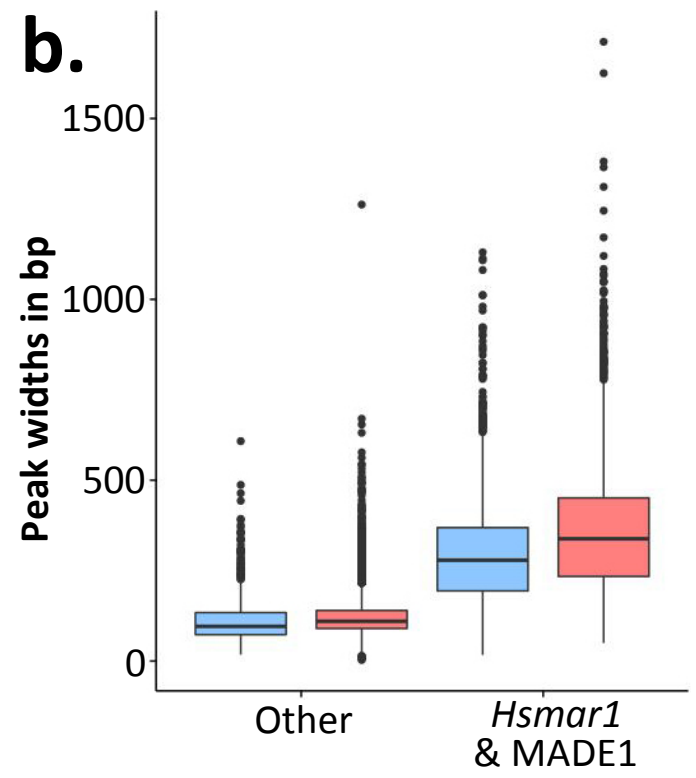

Targets colocalizing with peaks 
Antoine-Lorquin_Fig. 3

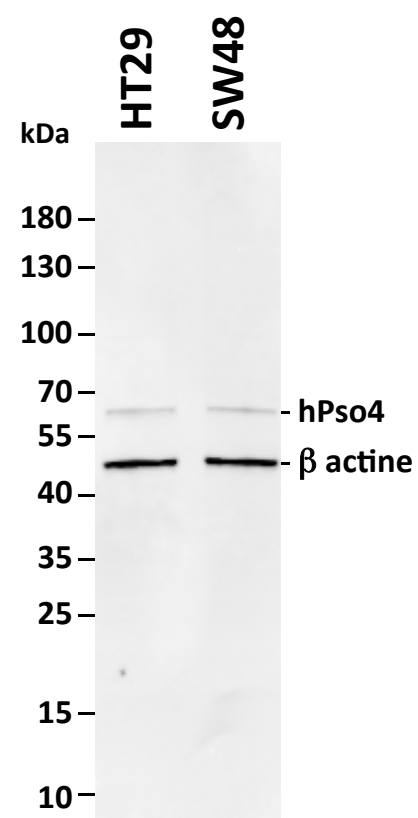




\section{Antoine-Lorquin_Fig.4}

a.

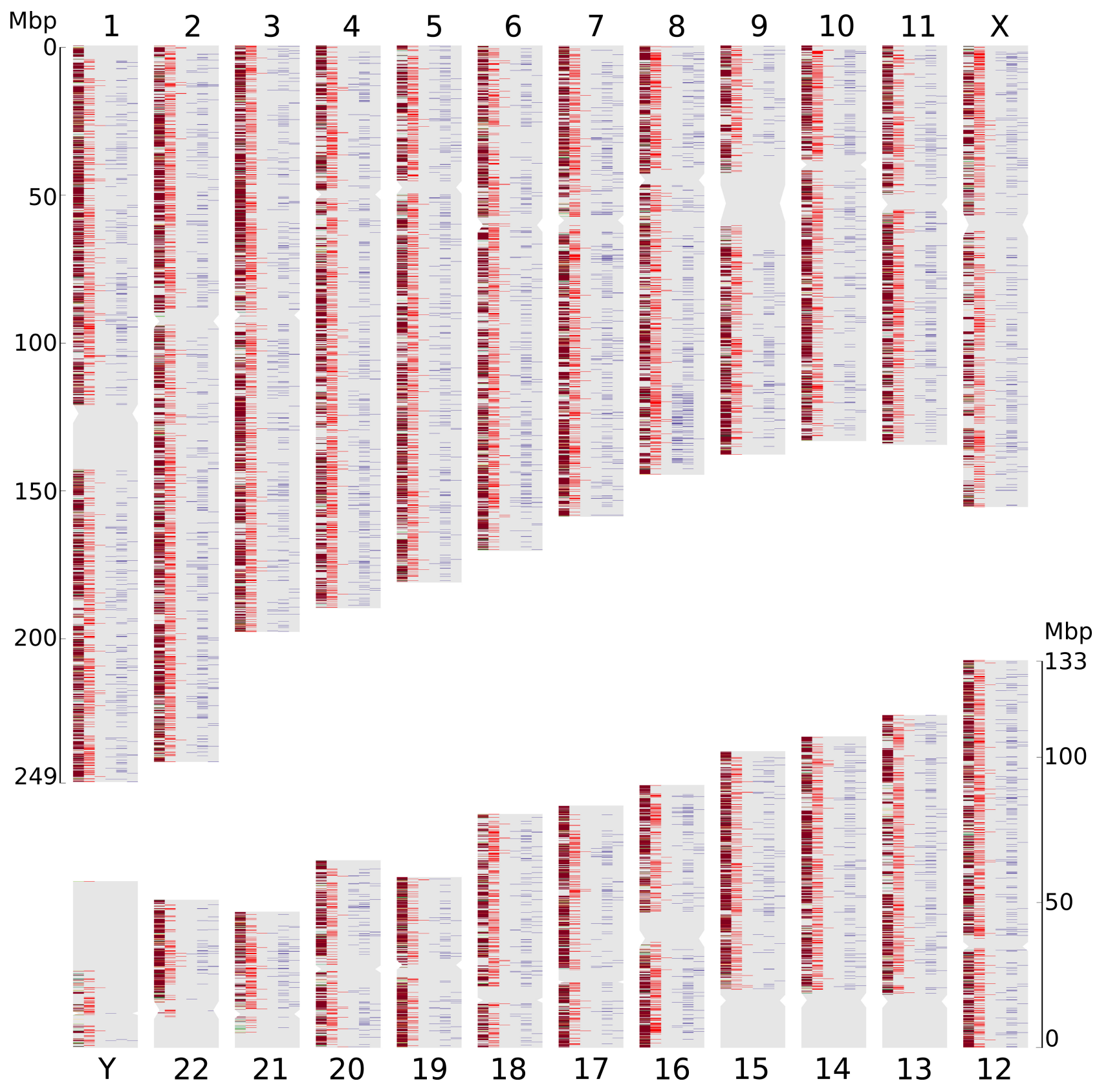


b.

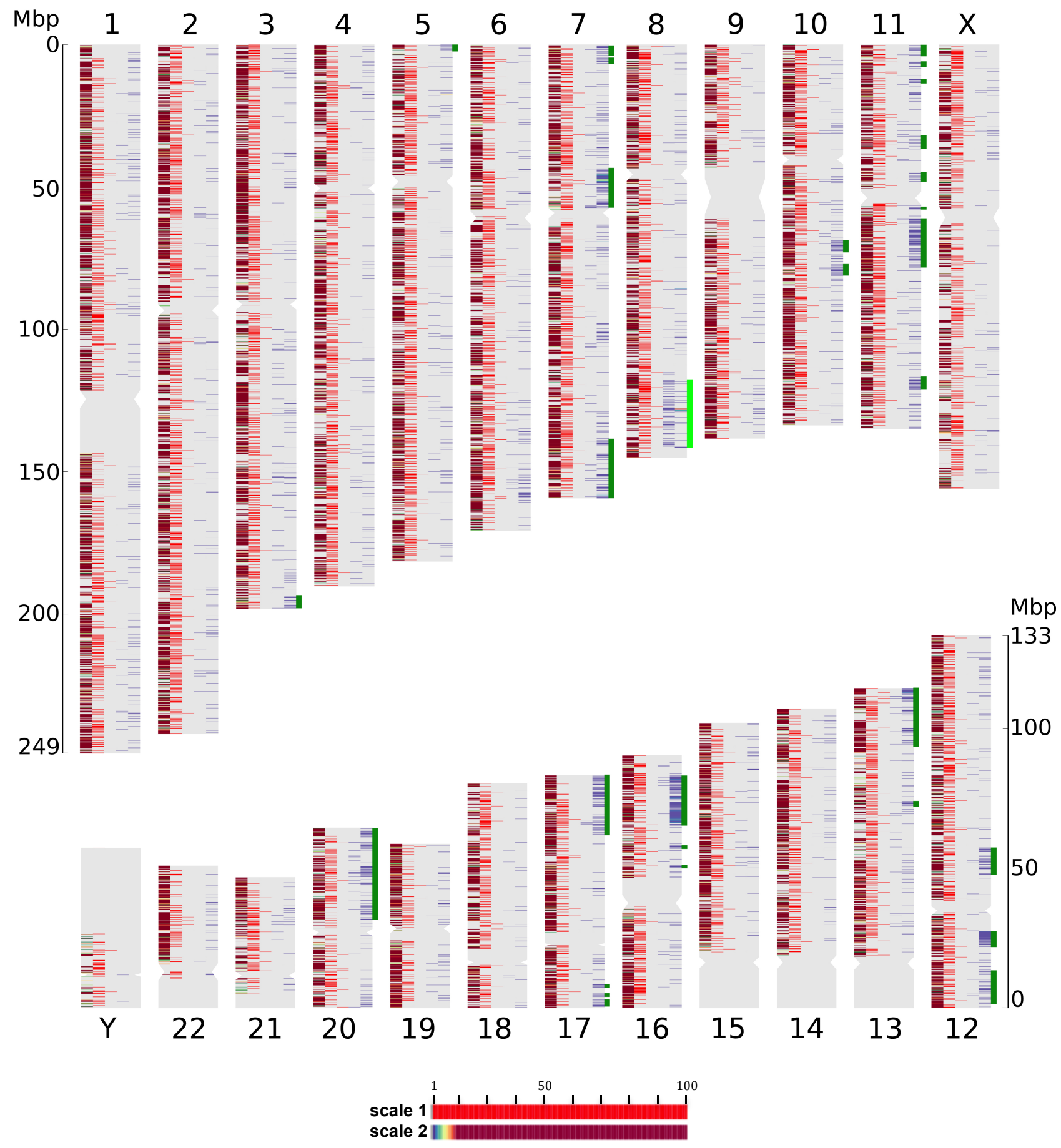


a.

$$
\begin{aligned}
& k D a \\
& V 2=62 \quad- \\
& V 3=55 \quad \\
& \text { X2 }=49.7- \\
& \text { HSMAR1 }=40-
\end{aligned}
$$$$
\begin{array}{llllll}
\text { H } & \text { T } & 1 & 2 & 3 & 4
\end{array}
$$

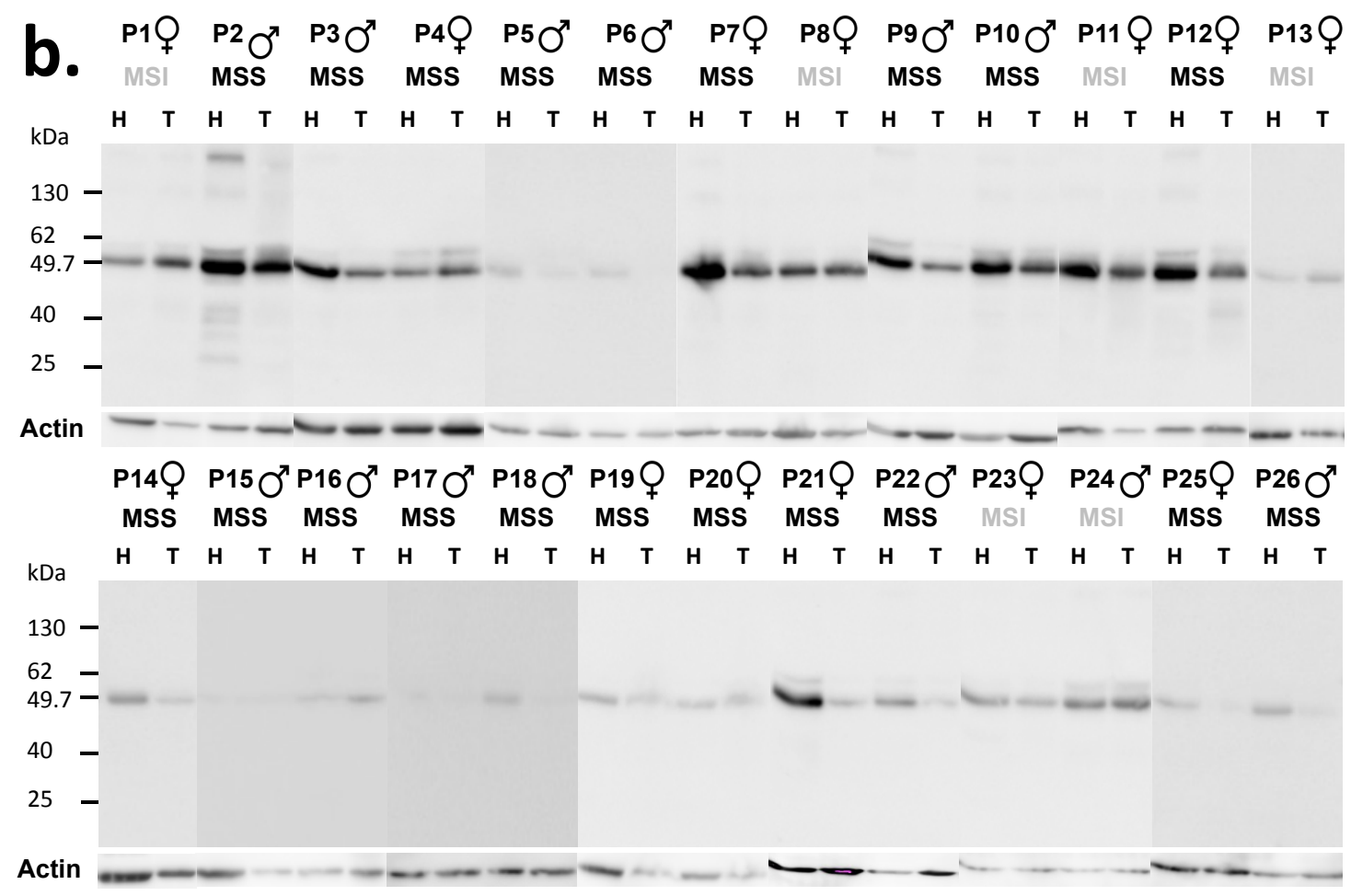

